\title{
The right ventricle after cardiopulmonary bypass: new insights on its adaptive physiology
}

\author{
Gabriele Via ${ }^{1} \cdot$ Guido Tavazzi ${ }^{2,3} \cdot$ Karim Bendjelid $^{4}$
}

Received: 25 February 2020 / Accepted: 5 March 2020 / Published online: 10 March 2020

(c) Springer Nature B.V. 2020

Post cardiopulmonary bypass (CPB) right ventricular (RV) dysfunction has been extensively described in cardiac surgery in past years, and attributed to either cardioplegia, myocardial hypothermia, cardiac stunning, and pericardiectomy [1-3]. More recently, both a different contraction pattern $[4,5]$ and a dissociation of RV output from RV longitudinal contraction [6] have been described in this context, leading to some re-interpretation of what previously observed.

In the present issue of the Journal of Clinical Monitoring and Computing Korshin et al. describe interesting echocardiographic observations which cast further light on the increasingly studied behaviour of the right ventricle (RV) after on-pump cardiac surgery [7]. By speckle tracking analysis of RV regional contraction and motion, the authors elegantly characterized the RV contractility pattern in different phases of uncomplicated CABG surgery, in patients with baseline normal biventricular function.

Their observational study analyses data acquired from a previous work of theirs. Regardless of the limitations the authors themselves acknowledge (mostly the retrospective nature of the study and the small population), their work has its strength in the rigorous methodology of the echocardiographic acquisition and analysis, and in the simultaneous assessment of macro-hemodynamics with the pulmonary artery catheter. The TEE views studied (A4Ch and transgastric modified RV inflow-outflow) take into account

Gabriele Via

gabriele.via@gmail.com

1 Cardiac Anesthesia and Intensive Care Dept., Fondazione Cardiocentro Ticino, Lugano, Switzerland

2 Anesthesia, Intensive Care and Pain Therapy, Department of Clinical, Surgical, Diagnostic and Pediatric Sciences, University of Pavia, Pavia, Italy

3 Anesthesia and Intensive Care, Emergency Dept., Fondazione IRCCS Policlinico S. Matteo, Pavia, Italy

4 Department of Anaesthesia, Pharmacology and Intensive Care, Geneva University Hospitals, Geneva, Switzerland the complex RV geometry, while invasive hemodynamic monitoring characterized the cardiovascular changes from anaesthesia induction to weaning from CPB and sternotomy closure.

Interestingly, while a constant reduction in longitudinal function from pre to post-bypass to chest closure (measured both as TAPSE, $\mathrm{S}^{\prime}$ and speckle tracking derived RV longitudinal displacement) was observed, in post-CPB both free wall and septum speckle tracking-derived transversal displacement increased, and contributed to preserving stroke volume (SV). Post chest closure SV reduction was then observed, due to both RV end-diastolic volume reduction, and loss of this "septal compensation" [7, 8]. However, as paradoxical septal motion (PSM) is a frequent echocardiographic finding after $\mathrm{CPB}$, we may speculate that all the septal features, observed in the present study, are related to the open chest-pericardium and their closure. Indeed, it is well known that PSM may occur whenever the pericardium is cut, as this structure restrains the anterior cardiac mobility resulting in forward motion of the entire heart in systole. Unfortunately, the surgical procedure has not been well documented to confirm the present hypothesis [9].

Throughout the entire study period, corresponding speckle-tracking strain measurements of the free wall and the septum remained unchanged, indicating a change in geometry and systolic function pattern, rather than a compensatory contractility increase, as mechanism for RV output preservation, despite longitudinal function reduction.

The RV, differently from the LV, usually works under its compliance limit having a wide degree of potential adaptation to altered preload and, to a lesser extent, afterload and variations [10]. Its range of systolic and motion pattern changes has been partially investigated in pulmonary hypertensive patients, where the chronic nature of the disease allowed the RV and pulmonary tree to gradually modify and adapt to the level of afterload increase [11].

Such observations are less studied in the acute cardiac surgery setting, where the variations of compartment status 
(open chest versus closed chest) are likely to highly influence volumetric and ventricular compliance conditions. The results of the study could be interpreted as a further step in the demonstration of RV adaptation capacity in different conditions.

$\mathrm{RV}$ strain emerges as a potentially more accurate tool for RV behaviour characterization and monitoring in this setting. This should be tested in the future also on patients with overt RV failure after CPB. Also it should be proved in comparison with the ease of use of traditional echocardiographic measures of RV function (such as right ventricular ejection fraction), and with the comprehensiveness of 3D echo.

The message reinforced by Korshin et al. is that assessment of RV function post cardiac surgery should not neglect the peculiar changes in its systolic function pattern. Relying solely on longitudinal contraction as a measure of global $\mathrm{RV}$ systolic function after cardiac surgery is highly prone to inaccuracy and overdiagnosis of RV dysfunction. Altogether, while in physiological conditions RV output is generated mainly by longitudinal contraction, we shouldn't forget that, after CPB, the RV should always be regarded as "a different animal", and as such assessed.

\section{Compliance with ethical standards}

Conflict of interest The authors declare that they have no conflict of interest.

\section{References}

1. Christakis GT, Buth KJ, Weisel RD, et al. Randomized study of right ventricular function with intermittent warm or cold cardioplegia. Ann Thorac Surg. 1996;61(1):128-34.
2. Unsworth B, Casula RP, Yadav H, et al. Contrasting effect of different cardiothoracic operations on echocardiographic right ventricular long axis velocities, and implications for interpretation of post-operative values. Int J Cardiol. 2013;165(1):151-60.

3. Zanobini M, Saccocci M, Tamborini G, et al. Postoperative echocardiographic reduction of right ventricular function: is pericardial opening modality the main culprit? Biomed Res Int. 2017;2017:4808757.

4. Raina A, Vaidya A, Gertz ZM, Susan C, Forfia PR. Marked changes in right ventricular contractile pattern after cardiothoracic surgery: implications for post-surgical assessment of right ventricular function. J Heart Lung Transplant. 2013;32(8):777-83.

5. Tamborini G, Muratori M, Brusoni D, et al. Is right ventricular systolic function reduced after cardiac surgery? A two- and three-dimensional echocardiographic study. Eur J Echocardiogr. 2009;10(5):630-4.

6. Korshin A, Gronlykke L, Nilsson JC, et al. Tricuspid annular plane systolic excursion is significantly reduced during uncomplicated coronary artery bypass surgery: a prospective observational study. J Thorac Cardiovasc Surg. 2019;158(2):480-9.

7. Korshin A, Gronlykke L, Holmgaard F, et al. Right ventricular transverse displacement increases following cardiac surgery: possibly compensating loss in tricuspid annular plane systolic excursion (TAPSE). J Clin Monit Comput. 2020. https://doi. org/10.1007/s10877-020-00466-2.

8. Bendjelid K. Right atrial pressure: determinant or result of change in venous return? Chest. 2005;128(5):3639-40.

9. Reynolds HR, Tunick PA, Grossi EA, Dilmanian H, Colvin SB, Kronzon I. Paradoxical septal motion after cardiac surgery: a review of 3,292 cases. Clin Cardiol. 2007;30(12):621-3.

10. Pinsky MR. The right ventricle: interaction with the pulmonary circulation. Crit Care. 2016;20:266.

11. Friedberg MK, Redington AN. Right versus left ventricular failure: differences, similarities, and interactions. Circulation. 2014;129(9):1033-44.

Publisher's Note Springer Nature remains neutral with regard to jurisdictional claims in published maps and institutional affiliations. 